\title{
On the Theory of Continued Fractions.
}

By Haripada Datta, Research Student, Edinburgh University.

(Read and Received 14th January 1916).

An analytic function is generally given either directly as a power-series, or at anyrate in some form which can readily be converted into a power-series. The power-series is not an altogether satisfactory method of representing the function, on account of the failure of its convergence outside its circle of convergence; in this respect it is decidedly inferior to the method of representing the function by a continued fraction, as this latter expression in many cases converges over the whole plane, except on certain curves. But it is not so easy to express the function as a continued fraction in the first place, and many of the known representations of functions as continued fractions are obtained by indirect artifices. In the present paper it is shown that for a large class of cases, including many of the important known functions, the continued-fraction-expression can be obtained directly and practically from the power series by using a known transformation which involves determinants, and evaluating these determinants. By this method a considerable number of known isolated results are connected together and exhibited as parts of a systematic theory: and some new results are obtained.

\section{PART I.}

In Part I. we shall deal with cases in which a function defined by a series of the form

$$
\frac{\alpha_{0}}{x}+\frac{\alpha_{1}}{x^{2}}+\frac{\alpha_{-2}}{x^{3}}+\ldots+\frac{\alpha_{n-1}}{x^{n}}+\ldots
$$

is to be converted into a continued fraction of the form

$$
\frac{a_{1}}{x+b_{1}}+\frac{a_{3}}{x+b_{2}}+\frac{a_{3}}{x+b_{3}}+\frac{a_{4}}{x+b_{4}}+\ldots
$$


That this problem reduces in all cases to a problem in determinants was shown first by Heilermann ${ }^{*}$; if the $n^{\text {th }}$ convergent to the continued fraction be denoted by $\phi_{n-1}(x) / f_{n}(x)$, we have

where

$$
f_{n}(x)=x^{n}-\frac{{ }^{1} K_{n}}{K_{n}} x^{n-1}+\frac{{ }^{2} K_{n}}{K_{n}} x^{n-2}-\ldots+(-1)^{n} \frac{K_{n}}{K_{n}}
$$

$$
K_{n+1}=\left|\begin{array}{llllll}
\alpha_{n} & \alpha_{n-1} & \alpha_{n-2} & \ldots & \alpha_{1} & \alpha_{0} \\
\alpha_{n+1} & \alpha_{n} & \alpha_{n-1} & \ldots & \alpha_{2} & \alpha_{1} \\
\alpha_{n+2} & \alpha_{n+1} & \alpha_{n} & \ldots & \alpha_{3} & \alpha_{2} \\
\ldots \ldots \ldots \ldots \ldots \ldots \ldots \ldots \ldots \ldots \ldots \ldots .1 & \ldots \ldots \ldots \ldots \ldots \ldots \\
\alpha_{2 n-1} & \alpha_{2 n-2} & \alpha_{2 n-3} & \ldots & \alpha_{n} & \alpha_{n-1} \\
\alpha_{2 n} & \alpha_{2 n-1} & \alpha_{2 n-2} & \ldots & \alpha_{n+1} & \alpha_{n}
\end{array}\right|
$$

and $K_{n}$ is obtained by deleting the first column and last row of this determinant, ${ }^{1} K_{n}$ is obtained by deleting the second column and last row, and so on.

Now the ordinary recurrence-formula between three successive convergents to a continued fraction gives in this case

$$
f_{n}(x)=\left(x+b_{n}\right) f_{n-1}(x)+a_{n} f_{n-2}(x) .
$$

Equating to zero the coefficient of $x^{n-1}$ and the constant term in this equation, we find

$$
\text { and } \begin{array}{rlrl}
b_{n} & =\frac{{ }^{1} K_{n-1}-{ }^{1} K_{n}}{K_{n}} \\
\text { or } & \frac{{ }^{n-2} K_{n-2}}{K_{n-2}} a_{n} & =\frac{{ }^{n} K_{n}}{K_{n}}+b_{n} \frac{{ }^{n-1} K_{n-1}}{K_{n-1}} \\
a_{n} & =\frac{K_{n} K_{n-2}}{K_{n-1}^{2}}
\end{array}
$$

The numerator of the $n^{\text {th }}$ convergent may be written

$$
\phi_{n-1}(x)=\gamma_{n-1} x^{n-1}+\gamma_{n-2} x^{n-2}+\ldots+\gamma_{1} x+\gamma_{0}
$$

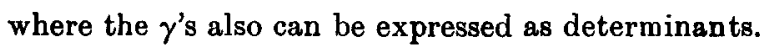

In what follows we shall transform into continued fractions the following series by evaluating the determinants which arise in the application of Heilermann's method. It will be shown that most

" Journal für Math. 33 (1845), p. 174. 
of the known representations of analytic functions as continued fractions are particular cases of the formulae thus derived.
(A) $\frac{1}{a} \frac{1}{x}+\frac{1}{a+d} \frac{1}{x^{2}}+\frac{1}{a+2 d} \frac{1}{x^{3}}+\ldots$
(B) $\frac{1}{a} \frac{1}{x}+\frac{1}{a(a+d)} \frac{1}{x^{2}}+\frac{1}{a(a+d)(a+2 d)} \frac{1}{x^{3}}+\ldots$
(C) $\frac{D}{a} \frac{1}{x}+\frac{D(D+d)}{a(a+d)} \frac{1}{x^{2}}+\frac{D(D+d)(D+2 d)}{a(a+d)(a+2 d)} \frac{1}{x^{3}}+\ldots$
(D) $\frac{1}{x}+\frac{a}{x^{2}}+\frac{a(a+d)}{x^{3}}+\frac{a(a+d)(a+2 d)}{x^{4}}+\ldots$
(E) $\frac{1}{\Pi(a, a+(r-1) d)} \frac{1}{x}+\frac{1}{\Pi(a+d, a+r d)} \frac{1}{x^{2}}$

$$
+\frac{1}{\Pi(a+2 d, a+(r+1) d)} \frac{1}{x^{3}}+\ldots
$$

where $\Pi(a, a+(r-1) d)$ denotes the product of $r$ factors beginning with the factor $a$ and ending with the factor $a+(r-1) d$, and the common difference between two consecutive factors is $d$.

$$
\frac{1}{\alpha+q^{a}} \frac{1}{x}+\frac{1}{\alpha+q^{a+d}} \frac{1}{x^{2}}+\frac{1}{\alpha+q^{a+2 d}} \frac{1}{x^{3}}+\ldots
$$

$$
\frac{1}{\alpha+q^{a}} \frac{1}{x}+\frac{1}{\left(\alpha+q^{a}\right)\left(\alpha+q^{a+\alpha}\right)} \frac{1}{x^{2}}+\ldots
$$

(H) $\frac{\alpha+q^{D}}{\alpha+q^{a}} \frac{1}{x}+\frac{\left(\alpha+q^{D}\right)\left(\alpha+q^{D+d}\right)}{\left(\alpha+q^{a}\right)\left(\alpha+q^{a+c}\right)} \frac{1}{x^{3}}+\ldots$

(L) $\frac{1}{x}+\frac{\alpha+q^{\alpha}}{x^{2}}+\frac{\left(\alpha+q^{a}\right)\left(\alpha+q^{\alpha+d}\right)}{x^{3}}+\ldots$

(M) $\frac{1}{\Pi\left(\alpha+q^{a}, \alpha+q^{a+(r-1) \alpha}\right)} \frac{1}{x}+\frac{1}{\Pi\left(\alpha+q^{a+d}, \alpha+q^{a+r d}\right)} \frac{1}{x^{2}}+\ldots$

(N) $q x+q^{4} x^{3}+q^{9} x^{3}+\ldots+q^{n^{2}} x^{n}+\ldots$

The determinants will be denoted according to the titles of the sections to which they belong, so that $A_{n}$ denotes the principal determinant in case (A).

By the operation $p$ row $_{s}+q$ row $_{t}=r$ rows $_{s}$, we shall imply that the elements of the $s^{\text {th }}$ row multiplied by $p$, added to the elewents of the $t^{\text {th }}$ row multiplied by $q$, form the elements of the new $s^{\text {th }}$ row, when a common factor $r$ has been taken out. 
(A).

In case (A) we shall show that the formulae for the elements of the continued fraction are

$$
\begin{aligned}
& a_{1}=\frac{1}{a} \\
& b_{n}=\frac{(n-1)\{a+(n-2) d\}}{a+(2 n-3) d}-\frac{n\{a+(n-1) d\}}{a+(2 n-1) d} \\
& a_{n}=-\frac{d^{2}(n-1)^{2}\{a+(n-2) d\}^{2}}{\{a+(2 n-2) d\}\{a+(2 n-3) d\}^{2}\{a+(2 n-4) d\}},
\end{aligned}
$$

so that

$$
\begin{gathered}
f_{n}=x^{n}-\frac{n}{1 !} \frac{a+(n-1) d}{a+(2 n-1) d} x^{n-1}+\frac{n(n-1)}{2 !} \\
\frac{\{a+(n-1) d\}\{a+(n-2) d\}}{\{a+(2 n-1) d\}\{a+(2 n-2) d\}} x^{n-2}-\ldots \\
+(-1)^{n} \frac{n !}{n !} \frac{\{a+(n-1) d\} \ldots(a+d) a}{\{a+(2 n-1) d\} \ldots(a+n d)} .
\end{gathered}
$$

The proof in this case is straightforward if we make use of a theorem due to Sir T. Muir, ${ }^{*}$ namely that

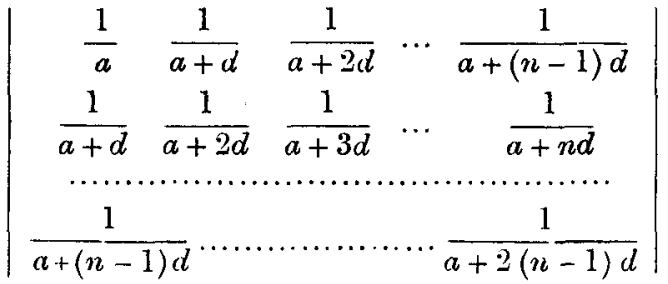

$$
\begin{aligned}
& =\frac{\left\{(n-1) ! d^{n-1} \cdot(n-2) ! d^{n-2} \ldots 2 ! d^{2} \cdot 1 ! d\right\}^{2}}{a(a+d)^{2}(a+2 d)^{3} \ldots\left\{a+(n-1) d_{\}}^{n}(a+n d)^{n-1} \ldots\{a+(2 n-2) d\}\right.} .
\end{aligned}
$$

From this we readily find

$$
\begin{aligned}
& \frac{{ }^{\prime} A_{n}}{\bar{A}_{n}}=\frac{n}{1 !} \frac{a+(n-1) d}{a+(2 n-1) d} \\
& \frac{{ }^{2} A_{n}}{A_{n}}=\frac{n(n-1)}{2 !} \frac{\left\{a+(n-1) d_{j}\{a+(n-2) d\}\right.}{\{a+(2 n-1) d\}\{a+(2 n-2) d\}} \\
& \frac{n A_{n}}{A_{n}}=\frac{n !}{n !} \frac{\{a+(n-1) d\} \ldots(a+d) a}{\{a+(2 n-1) d\} \ldots(a+n d)} .
\end{aligned}
$$

* Messenger of Muths. 36 (1906), p. 85. 
Ex. 1. $-\log (1+x)=x-\frac{x^{2}}{2}+\frac{x^{3}}{3}-\frac{x^{4}}{4}+\ldots$

$$
=\frac{2 x}{x+2}-\frac{x^{2}}{3(x+2)}-\frac{(2 x)^{2}}{5(x+2)}-\frac{(3 x)^{2}}{7(x+2)}-\cdots
$$

Ex. 2. $-2 \operatorname{coth}^{-1} x=\log \left(\frac{x+1}{x-1}\right)=\log \left(1+\frac{2}{x-1}\right)$

$$
\begin{aligned}
& =\frac{2}{x-1}-\frac{1}{2}\left(\frac{2}{x-1}\right)^{2}+\frac{1}{3}\left(\frac{2}{x-1}\right)^{3}-\cdots \\
& =\frac{2}{x}-\frac{1}{3 x}-\frac{2^{2}}{5 x}-\frac{3^{2}}{7 x}-\cdots
\end{aligned}
$$

$$
\text { Ex. 3.-2 } \begin{aligned}
\tanh ^{-1} x & =\log \left(\frac{1+x}{1-x}\right)=\log \left(1+\frac{2 x}{1-x}\right) \\
& =\frac{2 x}{1}-\frac{x^{2}}{3}-\frac{(3 x)^{2}}{7}-\frac{(4 x)^{2}}{9}-\cdots
\end{aligned}
$$

(B).

In case (B) we shall show that the elements of the continued fraction are given by the formulae

$$
\begin{aligned}
& a_{1}=\frac{1}{a} \\
& b_{1}=-\frac{1}{a+d} \\
& b_{n}=\frac{d-a}{\{a+(2 n-3) d\}\{a+(2 n-1) d\}} \\
& a_{n}=\frac{d(n-1)\{a+(n-1) d\}}{\{a+(2 n-4) d\}\{a+(2 n-3) d\}^{2}\{a+(2 n-2) d\}} . \\
& f_{n}=x^{n}-\frac{n}{1 !} \frac{1}{a+(2 n-1) d} x^{n-1}+\frac{n(n-1)}{2 !} \\
& \times \frac{1}{\{a+(2 n-1) d\}\{a+(2 n-2) d\}} x^{n-2}-\ldots \\
& \ldots+(-1)^{n} \frac{n !}{n !} \frac{1}{\{a+(2 n-1) d\} \ldots(a+n d)} .
\end{aligned}
$$


Proof :-

We have

$$
B_{4}=\left|\begin{array}{cccc}
\frac{1}{a \ldots(a+3 d)} & \frac{1}{a \ldots(a+2 d)} & \frac{1}{a(a+d)} & \frac{1}{a} \\
\frac{1}{a \ldots(a+4 \mathrm{~d})} & \frac{1}{a \ldots(a+3 d)} & \frac{1}{a \ldots(a+2 d)} & \frac{1}{a(a+d)} \\
\frac{1}{a \ldots(a+5 d)} & \frac{1}{a \ldots(a+4 d)} & \frac{1}{a \ldots(a+3 d)} & \frac{1}{a \ldots(a+2 d)} \\
\frac{1}{a \ldots(a+6 d)} & \frac{1}{a \ldots(a+5 d)} & \frac{1}{a \ldots(a+4 d)} & \frac{1}{a \ldots(a+3 d)}
\end{array}\right|
$$

Performing on this determinant the operations

$$
\text { (1) } \begin{aligned}
& d \operatorname{row}_{4}-\operatorname{row}_{3}=-\operatorname{row}_{4}^{\prime} \\
& 2 d \operatorname{row}_{4}^{\prime}-\operatorname{row}_{2}=-\operatorname{row}_{4}^{\prime \prime} \\
& 3 d \operatorname{row}_{4}^{\prime \prime}-\operatorname{row}_{1}=-\operatorname{row}_{4}^{\prime \prime \prime} \\
& \text { (2) } \quad d \operatorname{row}_{3}-\operatorname{row}_{2}=-\operatorname{row}_{3}^{\prime} \\
& 2 d \operatorname{row}_{3}^{\prime}-\operatorname{row}_{1}=-\operatorname{row}_{3}^{\prime \prime} \\
& \text { (3) } d \text { row }_{2}-\operatorname{row}_{1}=-\operatorname{row}_{3}^{\prime \prime},
\end{aligned}
$$

we get

$$
\begin{aligned}
& B_{4}=\frac{(-1)^{\frac{4 \times 3}{2}}}{d^{\frac{3 \times 4}{2}} 3 ! 2 ! 1 !} \frac{1}{\{a(a+d)(a+2 d)\}\{a(a+d)\} a} \\
& \left|\begin{array}{cccc}
\frac{1}{a+3 d} & \frac{1}{a+2 d} & \frac{1}{a+d} & \frac{1}{a} \\
\frac{1}{a+4 d} & \frac{1}{a+3 d} & \frac{1}{a+2 d} & \frac{1}{a+d} \\
\frac{1}{a+5 d} & \frac{1}{a+4 d} & \frac{1}{a+3 d} & \frac{1}{a+2 d} \\
\frac{1}{a+6 d} & \frac{1}{a+5 d} & \frac{1}{a+4 d} & \frac{1}{a+3 d}
\end{array}\right|
\end{aligned}
$$

The last determinant is $A_{4}$. 
Similarly we get

$$
\begin{aligned}
& B_{n}=\frac{(-1)^{\frac{n(n-1)}{2}}}{d^{\frac{n(n-1)}{2}}} \times \frac{1}{(n-1) !(n-2) ! \ldots 2 ! 1 !} \\
& \times \frac{1}{\{a+(n-2) d\}\{a+(n-3) d\}^{2}\{a+\langle n-4) d\}^{3} \ldots a^{n-1}} \times A_{n}, \\
& { }^{1} B_{n}=\left(-d^{-1}\right)^{\frac{n(n-1)}{2}} \frac{1}{(n-1) !(n-2) ! \ldots 2 ! 1 !} \\
& \times \frac{1}{\{a+(n-1) d\}\{a+(n-2) d\}\{a+(n-3) d\}^{2} \ldots a^{n-1}}{ }^{1} A_{n} \\
& { }^{n} B_{n}=\frac{\left(-d^{-1}\right)^{\frac{n(n-1)}{2}}}{(n-1) !(n-2) ! \ldots 2 ! 1 !\{a+(n-1) d\}\{a+(n-2) d\}^{2} \ldots a^{\prime \prime}}{ }^{*} A_{n} \text {. } \\
& E x .-e^{x}=1+x+\frac{x^{2}}{2 !}+\frac{x^{3}}{3 !}+\cdots \\
& =1+\frac{2 x}{1-x}+\frac{x^{2}}{6}+\frac{x^{2}}{10}+\frac{x^{2}}{14}+\cdots
\end{aligned}
$$

This ontinued fraction for the exponential function was discovered by Lagrange. *

In case (C) the formulae will be shown to be

$$
\begin{aligned}
& a_{1}=\frac{D}{a} \\
& b_{1}=-\frac{D+d}{a+d} \\
& b_{n}=\frac{(n-1)\{D+(n-1) d\}}{a+(2 n-3) d}-\frac{n(D+n d)}{a+(2 n-1) d} \\
& a_{n}=\frac{d(n-1)\{a+(n-2) d\}\{D+(n-1) d\}\{D-a-(n-2) d\}}{\{a+(2 n-2) d\}\{a+(2 n-3) d\}^{2}\{a+(2 n-4) d\}} \\
& f_{n}= x^{n}-\frac{n}{1 !} \frac{D+n d}{a+(2 n-1) d} x^{n-1}+\frac{n(n-1)}{2 !} \\
& \quad \times \frac{(D+n a)\{D+(n-1) d\}}{\{D+(2 n-1) d\}\{a+(2 n-2) d\}} x^{n-2}-\ldots \\
& \ldots+(-1)^{n} \frac{(D+n d)\{D+(n-1) d\} \ldots(D+d)}{\{a+(2 n-1) d\} \ldots \ldots \ldots \ldots(a+n d)} .
\end{aligned}
$$

* Works of Lagrange, 4, p. 319 . 


\section{Proof :-}

We have

$$
C_{4}=\left|\begin{array}{llll}
\frac{D \ldots(D+3 d)}{a \ldots(a+3 d)} & \frac{D \ldots(D+2 d)}{a \ldots(a+2 d)} & \frac{D(D+d)}{a(a+d)} & \frac{D}{a} \\
\frac{D \ldots(D+4 d)}{a \ldots(a+4 d)} & \frac{D \ldots(D+3 d)}{a \ldots(a+3 d)} & \frac{D \ldots(D+2 d)}{a \ldots(a+2 d)} & \frac{D(D+d)}{a(a+d)} \\
\frac{D \ldots(D+5 d)}{a \ldots(a+5 d)} & \frac{D \ldots(D+4 d)}{a \ldots(a+4 d)} & \frac{D \ldots(D+3 d)}{a \ldots(a+3 d)} & \frac{D \ldots(D+2 d)}{a \ldots(a+2 d)} \\
\frac{D \ldots(D+6 d)}{a \ldots(a+6 d)} & \frac{D \ldots(D+5 d)}{a \ldots(a+5 d)} & \frac{D \ldots(D+4 d)}{a \ldots(a+4 d)} & \frac{D \ldots(D+3 d)}{a \ldots(a+3 d)}
\end{array}\right|
$$

Let us perform on this determinant the operations

$$
\begin{aligned}
\text { (1) } \operatorname{row}_{4}-\operatorname{row}_{3} & =(D-a) \text { row }_{4}^{\prime} \\
\operatorname{row}_{3}-\operatorname{row}_{2} & =(D-a) \text { row }_{3}^{\prime} \\
\operatorname{row}_{2}-\operatorname{row}_{1} & =(D-a) \text { row }_{2}^{\prime} \\
\text { (2) } \operatorname{row}_{4}^{\prime}-\operatorname{row}_{3}^{\prime} & =(D-a-d) \text { row }_{4}^{\prime \prime} \\
\operatorname{row}_{3}^{\prime}-\operatorname{row}_{2}^{\prime} & =(D-a-d) \text { row }_{3}^{\prime \prime} \\
\text { (3) } \operatorname{row}_{4}^{\prime \prime}-\text { row }_{3}^{\prime \prime} & =(D-a-2 d) \text { row }_{4}^{\prime \prime} .
\end{aligned}
$$

Now the determinant

Thus we get

$$
\left|\begin{array}{l|}
D_{1} \\
D_{2}^{\prime} \\
D_{3}^{\prime \prime} \\
D_{4}^{\prime \prime \prime}
\end{array}\right| \text { is the same as } B_{4} \text {. }
$$

$$
C_{4}=\left[D^{4}(D+d)^{3}(D+2 d)^{2}(D+3 d)\right](D-a)^{3}(D-a-d)^{2}(D-a-2 d) B_{4} \text {. }
$$

Similarly, we get

$$
\begin{aligned}
& C_{n}=\left[D^{n}(D+d)^{n-1}(D+2 d)^{n-2} \ldots\{D+(n-1) d\}\right] \\
& \times\left[(D-a)^{n-1}(D-a-d)^{n-2} \ldots\{D-a-(n-2) d\}\right] b_{n} \\
& { }^{\mathrm{l}} C_{n}=\left[D^{n}(D+d)^{n-1} \ldots\{D+(n-2) d\}^{2}\{D+(n-1) d\}(D+n d)\right] \\
& \times\left[(D-a)^{n-1} \ldots \ldots\{\{D-a-(n-1) d\}] \times{ }^{1} B_{n}\right. \\
& { }^{n} C_{n}=\left[D^{n}(D+d)^{n}(D+2 d)^{n-1} \ldots(D+n d)\right] \\
& \times\left[(D-a)^{n-1} \ldots\{D-a-(n-2) d\}\right] \times{ }^{n} B_{n} .
\end{aligned}
$$


Ex. 1.- $\int_{l}^{m}\left(\frac{s-m}{l-s}\right)^{q} \frac{d s}{x-s}$

$$
\begin{aligned}
= & \frac{\Gamma(q+1) \Gamma(-q)}{1}\left[\frac{-q}{1 !} \frac{l-m}{l-x}+\frac{-q(-q+1)}{2 !}\left(\frac{l-m}{l-x}\right)^{2}\right. \\
& \left.\quad+\frac{-q(-q+1)(-q+2)}{3 !}\left(\frac{l-m}{l-x}\right)^{3}+\cdots\right] \\
= & \frac{\Gamma(q+1) \Gamma(1-q)}{\frac{l-x}{l-m}-\frac{1-q}{2}}+\frac{\frac{q^{2}-1}{4 \times 1 \times 3}}{\frac{l-x}{l-m}-\frac{1}{2}}+\frac{q^{2}-2^{2}}{\frac{4 \times 3 \times 5}{l-x}}+\cdots
\end{aligned}
$$

Ex. 2. $-\left(\frac{x-m}{x-l}\right)^{q}=\left(1+\frac{l-m}{x-l}\right)^{q}$

$$
=1+\frac{-2 q}{2\left(\frac{l-x}{l-m}\right)+q-1}+6 \frac{q^{2}-1}{6\left(\frac{l-x}{l-m}\right)-3}+\frac{q^{2}-2^{2}}{10\left(\frac{l-x}{l-m}\right)-5}+\cdots
$$

If $m=-1$ and $l=1$

then $\left(\frac{x+1}{x-1}\right)^{2}$ *

$$
=1+\frac{2 q}{x-q}+\frac{q^{2}-1}{3 x}+\frac{q^{2}-2^{2}}{5 x}+\frac{q^{2}-3^{2}}{7 x}+\cdots
$$

Ex. 3. $-(1+x)^{q}+=1+q x+\frac{q(q-1)}{2 !} x^{2}+\ldots$

$$
=1+\frac{2 q x}{(1-q) x+2}+\frac{\left(q^{2}-1^{2}\right) x^{2}}{3(x+2)}+\frac{\left(q^{2}-2^{2}\right) x^{2}}{5(x+2)}+\frac{\left(q^{2}-3^{2}\right) x^{2}}{7(x+2)}+\ldots
$$

$E x .4 \cdot-\frac{\sin ^{-1} x}{\sqrt{1-x^{2}}}=x+\frac{2}{3} x^{3}+\frac{2.4}{3.5} x^{5}+\frac{2.4 \cdot 6}{3.5 \cdot 7} x^{7}+\cdots$

$$
=\frac{x}{1}-\frac{1.2 x^{2}}{3}-\frac{1.2 x^{2}}{5}-\frac{3.4 x^{2}}{7}-\frac{3.4 x^{2}}{9}-\cdots
$$

Ex. 5. $-\left(1-x^{2}\right)^{\frac{1}{2}} \sin ^{-1} x=x-\frac{x^{3}}{3}-\frac{2}{3} \frac{x^{5}}{5}-\frac{2.4}{3 \cdot 5} \frac{x^{7}}{7}-\cdots$

$$
=x-\frac{x^{3}}{3}-\frac{2.3 x^{2}}{5}-\frac{2.3 x^{2}}{7}-\frac{4.5 x^{2}}{9}-\frac{4.5 x^{2}}{11}-\cdots
$$

* Laguerre, Bull. de la Société Math. de France, 8 (1879-80).

† Works of Lagrange, 4, p. 318. 
(D).

In case (D) the formulae for the elements of the continued fraction will be shown to be

$a_{1}=1$

$b_{1}=-a$

$b_{n}=-\{a+(2 n-2) d\}$

$a_{n}=-(n-1)\{a+(n-2) d\} d$.

$f_{n}=x^{n}-\frac{n}{1 !}\{a+(n-1) d\} x^{n-1}+\frac{n(n-1)}{2 !}\{a+(n-1) d\}$

Proof:-

$$
\begin{aligned}
& \times\{a+(n-2) d\} x^{n-2}-\ldots \\
& \ldots+(-1)^{n} \frac{n !}{n !}\{a+(n-1) d\} \ldots(a+d) a .
\end{aligned}
$$

We have $D_{n}=\{a(a+d)(a+2 d)\}\{a(a+d)\} a$

$\times\left|\begin{array}{cccc}1 & 1 & 1 & 1 \\ a+3 d & a+2 d & a+d & a \\ (a+3 d)(a+4 d) & (a+2 d)(a+3 d) & (a+d)(a+2 d) & a(a+d) \\ (a+3 d) \ldots(a+5 d) & (a+2 d) \ldots(a+4 d) & (a+d) \ldots(a+3 d) & a \ldots(a+2 d)\end{array}\right|$

On this determinant let us perform the operations :-

(1) row $_{4}-2 d$ row $_{3}=$ row $_{4}^{\prime}$

$$
\text { row }_{3}-d \text { row }_{3}=\text { row }_{3}^{\prime}
$$

(2) row $_{4}^{\prime}-d$ row $_{3}^{\prime}=$ row $_{4}^{\prime \prime}$.

Hence we get $D_{4}=a^{3}(a+d)^{2}(a+2 d)$

$$
\times\left|\begin{array}{cccc}
1 & 1 & 1 & 1 \\
a+3 d & a+2 d & a+d & a \\
(a+3 d)^{2} & (a+2 d)^{2} & (a+d)^{2} & a^{2} \\
(a+3 d)^{3} & (a+2 d)^{3} & (a+d)^{3} & a^{3}
\end{array}\right|
$$

This last determinant is a case of Vandermonde's.

Similarly we get

$$
\begin{aligned}
& \dot{D}_{n}=(-d)^{\frac{n(n-1)}{2}} a^{n-1}(a+d)^{n-2} \ldots\{a+(n-3) d\}^{2}\{a+(n-2) d\} \\
& \times(n-1) !(n-2) ! \ldots 2 ! 1 \text {. } \\
& { }^{1} D_{n}=(-d)^{\frac{n(n-1)}{2}} a^{n-1}(a+d)^{n-2} \ldots\{a+(n-3) d\}^{2}\{a+(n-2) d\}\{a+(n-1) d\} \\
& \times n !(n-2) ! \ldots 2 ! 1 \text {. } \\
& { }^{n} D_{n}=(-d)^{\frac{n(n-1)}{2}} a^{n}(a+d)^{n-1} \ldots\{a+(n-1) d\} \\
& \times(n-1) !(n-2) ! \ldots 2 ! 1 \text {. }
\end{aligned}
$$


$\begin{aligned} E x .1 .-\int_{x}^{\infty} \frac{e^{-x}}{x^{a}} d x & =\frac{e^{-x}}{x^{a-1}}\left\{\frac{1}{x}-\frac{a}{x^{2}}+\frac{a(a+1)}{x^{3}}-\cdots\right\} \\ & =\frac{e^{-x} x^{1-a}}{x+a}-\frac{a}{x+a+2}-\frac{2(a+1)}{x+a+4}-\frac{3(a+2)}{x+a+6}-\cdots\end{aligned}$

Ex. 2. $-\int_{x}^{\infty} e^{-x^{2}} d x^{*}=e^{-x^{2}} \frac{x}{2}\left\{\frac{1}{x^{2}}-\frac{\frac{1}{2}}{x^{4}}-\frac{\frac{1}{2} \cdot \frac{3}{2}}{x^{6}}-\cdots\right\}$

$$
=\frac{x e^{-x^{2}}}{2 x^{2}+1}-\frac{2}{2 x^{2}+5}-\frac{2(2 \times 3)}{2 x^{2}+9}-\frac{2(3 \times 5)}{2 x^{2}+13}-\frac{2(5 \times 7)}{2 x^{2}+17}-\cdots
$$

$E x$, s. $-\int_{x}^{\infty} e^{-x^{p}} d s=\frac{1}{p} \int_{y}^{\infty} \frac{e^{-t}}{t\left(1-\frac{1}{p}\right)} d t$ (where $t=s^{p}$ and $y=x^{p}$ )

$$
=\frac{\frac{x}{p} e^{-x^{p}}}{x^{p}+1-\frac{1}{p}}-\frac{1-\frac{1}{p}}{x^{p}+3-\frac{1}{p}}-\frac{2\left(1-\frac{1}{p}\right)}{x^{p}+5-\frac{1}{p}}-\cdots
$$

$E x .4 \cdot-\int_{x}^{\infty} \frac{e^{-\beta}}{s^{\alpha}} d s=\frac{1}{\beta} \int_{y}^{\infty} \frac{e^{-t}}{\frac{a+\beta-1}{\beta}} d t \quad$ (where $t=s^{\beta}, y=x^{\beta}$ )

$$
=\frac{\frac{1}{\beta} e^{-x \beta} x^{1-\alpha}}{x^{\beta}+\frac{a+\beta-1}{\beta}}-\frac{\frac{a+\beta-1}{\beta}}{x^{\beta}+\frac{a+3 \beta-1}{\beta}}-\frac{\frac{2(\alpha+2 \beta-1)}{\beta}}{x^{\beta}+\frac{a+5 \beta-1}{\beta}}-\cdots
$$

Ex. 5. $-\int_{0}^{\infty} \frac{e^{-x} x^{a-1}}{z+x} d x+=\Gamma(a)\left\{\frac{1}{z}-\frac{a}{z^{2}}+\frac{a(a+1)}{z^{3}}-\cdots\right\}$

$$
=\frac{\Gamma(a)}{z+a}-\frac{a}{z+a+2}-\frac{2(a+1)}{z+a+4}-\cdots
$$

(E).

In case (E) the formulae for the elements of the C.F. will be shown to be

$$
\begin{aligned}
& a_{\mathrm{t}}=\frac{1}{\Pi(a, a+(r-1) d} \\
& b_{n}=\frac{n-1}{a+(r+2 n-4) d}-\frac{n\{a+(n-1) d\}}{a+(r+2 n-2) d} \\
& a_{n}=-\frac{(n-1) d^{2}(r+n-2)\{a+(r+n-3) d\}\{a+(n-2) d\}}{\{a+(r+2 n-5) d\}\{a+(r+2 n-4) a\}^{2}\{a+(r+2 n-3) d\}}
\end{aligned}
$$

- This is the contracted form of Laplace's C.F.

† Stieltjes, oontracted form, Ann. Toulouse, 9 (1895). 


$$
\begin{aligned}
f_{n}=x^{n}- & \frac{n}{1 !} \frac{a+(n-1) d}{a+(2 n+r-2) d} x^{n-1} \\
& +\frac{n(n-1)}{2 !} \frac{\{a+(n-1) d\}\{a+(n-2) d\}}{\{a+(2 n+r-2) d\}\{a+(2 n+r-3) d\}} x^{n-2}-\ldots \\
& \ldots+(-1)^{n} \frac{\Pi(a+(n-1) d, a)}{\Pi(a+(2 n+r-2) d, a+(r+n-1) d)}
\end{aligned}
$$

Proof :-

Performing on the determinant $E_{4}$ the operations

$$
\begin{aligned}
& \text { (1) } \operatorname{row}_{4}-\text { row }_{3}=-r d \text { row }_{4}^{\prime} \\
& \text { row }_{3}-\text { row }_{2}=-r d \text { row }_{3}^{\prime} \\
& \text { row }_{2}-\text { row }_{1}=-r d \text { row }_{2}^{\prime} \\
& \text { (2) } \operatorname{row}_{4}^{\prime}-\text { row }_{3}^{\prime}=-(r+1) d \text { row }_{4}^{\prime \prime} \\
& \text { row }_{3}^{\prime}-\text { row }_{2}^{\prime}=-(r+1) d \text { row }_{3}^{\prime \prime} \\
& \text { (3) } \text { row }_{4}{ }^{\prime \prime}-\text { row }_{3}{ }^{\prime \prime}=-(r+2) d \text { row }_{4}^{\prime \prime} \\
& \text { (4) }-d \text { row }_{4} \text { "' }^{\prime \prime}+\text { row }_{3}{ }^{\prime \prime}=\text { row }_{4}{ }^{\text {iv }} \\
& -2 d \text { row }_{4}{ }^{\text {iv }}+\text { row }_{2}{ }^{\prime}=\text { row }_{4}{ }^{\prime} \\
& -3 d \text { row }_{4}{ }^{v}+\text { row }_{1} \\
& \text { (5) }-d \text { row }_{3}{ }^{\prime \prime}+\text { row }_{2}{ }^{\prime}=\text { row }_{3}^{\prime \prime \prime} \\
& -2 d \text { row }_{3}^{\prime \prime \prime}+\text { row }_{1} \\
& \text { (6) }-d \text { row }_{2}^{\prime}+\text { row }_{1} \text {, }
\end{aligned}
$$

we get

$$
\begin{aligned}
E_{4}=\frac{r^{3}(r+1)^{2}(r+2)}{3 ! 2 ! 1 ! \Pi(a, a+(r-2) d) \Pi(a+d, a+(r-1) d) \ldots \Pi(a+3 d, a+(r+1) d)} \\
\quad\left|\begin{array}{cccc}
\frac{1}{a+(r+2) d} & \frac{1}{a+(r+1) d} & \frac{1}{a+r d} & \frac{1}{a+(r-1) d} \\
\frac{1}{a+(r+3) d} & \frac{1}{a+(r+2) d} & \frac{1}{a+(r+1) d} & \frac{1}{a+r d} \\
\frac{1}{a+(r+4) d} & \frac{1}{a+(r+3) d} & \frac{1}{a+(r+2) d} & \frac{1}{a+(r+1) d} \\
\frac{1}{a+(r+5) d} & \frac{1}{a+(r+4) d} & \frac{1}{a+(r+3) d} & \frac{1}{a+(r+2) d}
\end{array}\right| .
\end{aligned}
$$

If we put $a$ for $a+(r-1) d$ in this determinant, it becomes $A_{4}$. This determinant is to be denoted by $A_{4}^{\prime}$. 
By a precisely similar process it can be shown that

$$
\begin{aligned}
E_{n}= & \frac{r^{n-1}(r+1)^{n-2}(r+2)^{n-3} \ldots(r+n-2)}{(n-1) !(n-2) !(n-3) ! \ldots 2 !} \\
& \times \frac{1}{\Pi(a, a+(r-2) d) \ldots \Pi(a+(n-1) d, a+(r+n-3) d)} A_{n}^{\prime} \\
{ }^{1} E_{n}= & \frac{r^{n-1}(r+1)^{n-2} \ldots(r+n-2)}{(n-1) !(n-2) ! \ldots 2 !}
\end{aligned}
$$

$\times \frac{1}{\Pi(a, a+(r-2) d) \ldots \Pi(a+(n-2) d, a+(r+n-4) d) \Pi(a+n d, a+(r+n-2) d)}:$

$$
\begin{aligned}
{ }^{n} E_{n}= & \frac{r^{n-1}(r+1)^{n-2}(r+n-2)}{(n-1) !(n-2) ! \ldots 2 !} \\
& \times \frac{1}{\Pi(a+d, a+(r-1) d) \ldots \Pi(a+n d, a+(r+n-2) d)}{ }^{n} A_{n}^{\prime} .
\end{aligned}
$$

In case (F) we shall show that

$$
\begin{aligned}
& a_{1}=\frac{1}{\alpha+q^{a}} \\
& b_{n}=\frac{q^{(n-1) d}-1}{q^{d}-1} \frac{\alpha+q^{a+(n-2) d}}{\alpha+q^{a+(2 n-3) d}}-\frac{q^{n d}-1}{q^{d}-1} \frac{\alpha+q^{a+(n-1) d}}{\alpha+q^{a+(2 n-1) d}} \\
& a_{n}=\frac{\alpha\left(q^{(n-1) d}-1\right)^{2}\left(\alpha+q^{a+(n-2) d}\right) q^{a+(2 n-4) d}}{\left(\alpha+q^{a+(2 n-4) d}\right)\left(\alpha+q^{a+(2 n-3) d}\right)^{2}\left(\alpha+q^{a+(2 n-2) d}\right)} \\
& f_{n}=x^{n}-\frac{q^{n d}-1}{q^{d}-1} \frac{q^{\frac{(n-1) n}{2} d}}{q^{\frac{(n-1) n}{2} d}} \frac{\alpha+q^{a+(n-1) d}}{\alpha+q^{a+(2 n-1) d}} x^{n-1}+\frac{\left(q^{n d}-1\right)\left(q^{(n-1) d}-1\right)}{\left(q^{d}-1\right)\left(q^{2 d}-1\right)} \\
& \quad \times \frac{q^{\frac{(n-1) n}{2} d}}{q^{\frac{(n-2)(n+1)}{2} d}} \frac{\left(\alpha+q^{a+(n-1) d}\right)\left(\alpha+q^{a+(n-2) d}\right)}{\left(\alpha+q^{a+(3 n-1) d}\right)\left(\alpha+q^{a+(2 n-2) d)}\right)} x^{n-2}-\ldots \\
& \quad \ldots+(-1)^{n} \frac{\left(\alpha+q^{a+(n-1) a}\right) \ldots\left(\alpha+q^{\alpha}\right)}{\left(\alpha+q^{a+(2 n-1) d}\right) \ldots\left(\alpha+q^{a+n d}\right)} \frac{q^{\frac{n(n-1)}{2} d}}{1}
\end{aligned}
$$

9 Vol. 34 
Proof :-In $F_{4}$, subtracting the last row from each of the rows above it and taking out common factors, and then subtracting the first column from each of the other columns, we get

$$
F_{4}=\frac{\alpha^{3}\left\{\left(q^{3 d}-1\right)\left(q^{2 a}-1\right)\left(q^{a}-1\right)\right\}^{2} q^{a+(a+d)+(a+2 d)} q^{d+2 \alpha}}{\left(\alpha+q^{a+6 d}\right)\left(\alpha+q^{a+3 d}\right)^{2}\left(\alpha+q^{a+d d}\right)^{2}\left(\alpha+q^{\alpha+3 d}\right)^{2}} F_{3}
$$

In a similar way we get

$$
\begin{aligned}
& \frac{1}{F_{n}} F_{n}=\frac{q^{n d}-1}{q^{d}-1} \frac{\alpha+q^{a+(n-1) d}}{\alpha+q^{\alpha+(2 n-1) d}} \times \frac{q^{\frac{(n-1) n}{2} d}}{q^{\frac{(n-1) n}{2} d}} \\
& \frac{{ }^{2} F_{n}}{F_{n}}=\frac{\left(q^{n d}-1\right)\left(q^{(n-1) d}-1\right)}{\left(q^{d}-1\right)\left(q^{2 l}-1\right)} \frac{\left(\alpha+q^{a+(n-1) d}\right)\left(\alpha+q^{a+(n-2) d}\right)}{\left(\alpha+q^{\alpha+(2 n-1) d}\right)\left(\alpha+q^{n+(2 n-2) d}\right)} \frac{q^{\frac{(n-1) n}{2} d}}{q^{\frac{(n-2)(n+1)}{2} d}}
\end{aligned}
$$$$
\frac{{ }^{n} F_{n}}{F_{n}}=\frac{\left(\alpha+q^{a+(n-1) d}\right) \ldots\left(\alpha+q^{a}\right)}{\left(\alpha+q^{a+(2 n-1) d}\right) \ldots\left(\alpha+q^{a+n d}\right)} \frac{q^{\frac{n(n-1)}{2} d}}{1} .
$$

In case $(G)$ we shall show that

$$
\begin{aligned}
& a_{1}=\frac{1}{\alpha+q^{\alpha}} \\
& b_{n}=\frac{q^{(n-1) d}}{q^{l}-1} \frac{1}{\alpha+q^{n+(2 n-3) d}}-\frac{q^{n d}-1}{q^{d}-1} \frac{1}{\alpha+q^{\alpha+(3 n-1) d}} \\
& a_{n}=\frac{q^{(2 n-3) d} q^{a+(n-3) d}\left(q^{(n-1) d}-1\right)\left(\alpha+q^{a+(n-2) d}\right)}{\left(\alpha+q^{n+(2 n-2) d}\right)\left(\alpha+q^{a+(2 n-3), d}\right)^{2}\left(\alpha+q^{a+(2 n-4) d}\right)} \\
& f_{n}=x^{n}-\frac{q^{n ! l}-1}{q^{d}-1} \frac{q^{\frac{(n-1) n}{2} d}}{q^{\frac{n(n-1)}{2} d}} \frac{1}{\alpha+q^{(2+(2 n-1) d}} x^{n-1}+\frac{\left(q^{n d}-1\right)\left(q^{(n-1) d}-1\right)}{\left(q^{d}-1\right)\left(q^{2 d}-1\right)} \\
& \times \frac{q^{\frac{(n-1) n}{2} d}}{q^{\frac{(n-2)(n+1)}{2} d}} \frac{1}{\left(\alpha+q^{n+(2 n-1) d}\right)\left(\alpha+q^{n+(2 n-2) d}\right)} x^{n-2}-\ldots \\
& \ldots+(-1)^{n} \frac{q^{\frac{(n-1) n}{2}} d}{1} \frac{1}{\left(\alpha+q^{a+(2 n-1) d}\right) \ldots\left(\alpha+q^{n i l}\right)} .
\end{aligned}
$$




\section{3}

Proof:-

Performing on the determinant $G_{4}$ the operations

we get

(1) $\alpha\left(q^{a}-1\right)$ row $_{4}+$ row $_{3}=q^{a}$ row ${ }_{4}^{\prime}$

$\alpha\left(q^{2 u}-1\right)$ row $_{4}{ }^{\prime \prime}+$ row $_{\mathrm{g}}=q^{9 u}$ row $_{4}{ }^{\prime \prime}$

$x\left(q^{3 d}-1\right)$ row $_{4}^{\prime \prime}+$ row $_{1}=q^{3 d}$ row $_{4}^{\prime \prime \prime}$;

(2) $\alpha\left(q^{a}-1\right)$ row $_{3}+$ row $_{2}=q^{d}$ row $_{3}^{\prime}$

$\alpha\left(q^{2 d}-1\right)$ row $_{3}{ }^{\prime}+$ row $_{1}=q^{2 d}$ row $_{3}{ }^{\prime \prime}$;

(3) $\alpha\left(q^{d}-1\right)$ row $_{2}+$ row $_{1}=q^{d}$ row $_{2}^{\prime}$

$$
\begin{gathered}
G_{+}=\frac{1}{\left(\alpha+q^{a}\right)^{3}\left(\alpha+q^{a+d}\right)^{2}\left(\alpha+q^{a+2 d}\right) \alpha^{3}\left(q^{d}-1\right)\left(q^{2 d}-1\right)\left(q^{3 a}-1\right)} \\
\times \frac{q^{a(1+2)}}{\alpha^{2}\left(q^{d}-1\right)\left(q^{2 d}-1\right)} \frac{q^{d(1+2+3)}}{\alpha\left(q^{d}-1\right)} F_{4} .
\end{gathered}
$$

In a similar way we can show that

$$
\begin{aligned}
G_{n}=\frac{q^{\frac{(n-1) n(n+1)}{b}}}{a^{\frac{(n-1) n}{2}}} & \times \frac{1}{\left(q^{d}-1\right)^{n-1}\left(q^{2, d}-1\right)^{n-2} \ldots\left(q^{(n-1) d}-1\right)} \\
& \times \frac{1}{\left(\alpha+q^{a}\right)^{n-1} \ldots\left(\alpha+q^{a+(n-2) d}\right)} F_{n} .
\end{aligned}
$$

In case $(H)$ we shall show that

$$
\begin{aligned}
& a_{1}=\frac{a+q^{D}}{\alpha+q^{a}} \\
& b_{n}=\frac{q^{(n-1) d}-1}{q^{d}-1} \frac{\alpha+q^{D+(n-1) d}}{q^{d}-1}-\frac{q^{n d}-1}{q^{d}-1} \frac{\alpha+q^{D+n d}}{\alpha+q^{a+(2 n-1) d}} \\
& a_{n}=\frac{\alpha q^{(2 n-3) d}\left(q^{(n-1) d}-1\right)\left(\alpha+q^{a+(u-2) d}\right)\left(\alpha+q^{D+(n-1) d}\right)\left(q^{a+(n-2) d}-q^{D}\right)}{\left(\alpha+q^{a+(2 n-1) d}\right)\left(\alpha+q^{(n+(2 n-3) d}\right)^{2}\left(\alpha+q^{a+(2 n-2) d}\right)} \\
& f_{n}=x^{n}-\frac{q^{\prime u d}-1}{q^{d}-1} \frac{q^{\frac{(n-1) n}{2} d}}{q^{\frac{(n-1) n}{2} d}} \frac{\alpha+q^{D_{+n d}}}{\alpha+q^{a+(2 n-1) d}} x^{n-1}+\frac{\left(q^{n d}-1\right)\left(q^{(n-1) d}-1\right)}{\left(q^{d}-1\right)\left(q^{2 d}-1\right)} \\
& \times \frac{q^{\frac{(n-1) n}{2} d}}{q^{\frac{(n-2)(n+1)}{2}} d} \frac{\left(\alpha+q^{D+(n-1) d}\right)\left(\alpha+q^{D+n d}\right)}{\left(\alpha+q^{i+(2 n-1 / d}\right)\left(\alpha+q^{a+(2 n-2) d}\right)} x^{n-2}-\ldots \\
& \ldots+(-1)^{n} \frac{q^{\frac{n(n-1)}{2} d}}{1} \frac{\left(\alpha+q^{D+d}\right) \ldots\left(\alpha+q^{D+n d}\right)}{\left(\alpha+q^{a+(2 n-1) d}\right) \ldots\left(\alpha+q^{n d}\right)} .
\end{aligned}
$$




\section{Proof :-}

Performing on $H_{4}$ the operations

we get

$$
\begin{aligned}
& \text { row }_{4}-\text { row }_{3}=\left(q^{D}-q^{a}\right) \text { row }_{4}^{\prime} \\
& -q^{a} \text { row }_{4}^{\prime}+\text { row }_{3}=\alpha \text { row }_{4}^{\prime \prime} \\
& \text { row }_{3}-\text { row }_{2}=\left(q^{D}-q^{a}\right) \text { row }_{3}^{\prime} \\
& -q^{a} \text { row }_{3}{ }^{\prime}+\text { row }_{2}=\alpha \text { row }_{3}^{\prime \prime} \\
& \text { row }_{2}-\text { row }_{1}=\left(q^{D}-q^{a}\right) \text { row }_{2}^{\prime} \\
& -q^{a} \text { row }_{2}^{\prime}-\text { row }_{1}=\alpha \text { row }_{2}^{\prime \prime} \\
& \text { row }_{4}{ }^{\prime \prime}-\text { row }_{3}{ }^{\prime \prime}=\left(q^{D}-q^{a+d}\right) \text { row }_{4}{ }^{\prime \prime} \\
& -q^{a+a l} \text { row }_{4} " \prime+\text { row }_{3} "=\alpha \text { row }_{4}{ }^{2 v} \\
& \text { (5) } \text { row }_{3}{ }^{\prime \prime}-\text { row }_{2}{ }^{\prime \prime}=\left(q^{D}-q^{a+d}\right) \text { row }_{3}{ }^{\prime \prime \prime} \\
& -q^{a+d} \text { row }_{3}^{\prime \prime \prime}+\text { row }_{2}^{\prime \prime}=\alpha \text { row }_{3}^{i v} \\
& \text { (6) } \quad \operatorname{row}_{4}^{\text {iv }}-\operatorname{row}_{3}^{\text {iv }}=\left(q^{D}-q^{a+2 d}\right) \operatorname{row}_{4}^{\text {iv }} \\
& -q^{\alpha+2 d} \text { row }_{4}{ }^{\mathrm{v}}+\text { row }_{3}{ }^{\mathrm{iv}}=\alpha \text { row }_{4}{ }^{\mathrm{vi}} \text {, }
\end{aligned}
$$

$$
\begin{aligned}
H_{4}=\left(\alpha+q^{D}\right)^{4}\left(\alpha+q^{D+d}\right)^{3}\left(\alpha+q^{D+2 d}\right)^{2}\left(\alpha+q^{D+3 d}\right) \\
\quad \times \frac{(-\alpha)^{\frac{3 x^{4}}{2}}\left(q^{D}-q^{a}\right)^{3}\left(q^{D}-q^{a+d}\right)^{2}\left(q^{D}-q^{a+2 d}\right)}{\left(q^{a}\right)^{3}\left(q^{a+d}\right)^{2} q^{a+2 d}} G_{4} .
\end{aligned}
$$

In case (L) we shall show that the elements of the C.F. are given by the equations

$$
\begin{aligned}
& a_{1}=1 \\
& b_{n}=\left(\alpha+q^{a+(n-2) d}\right) \frac{q^{(n-1) d}-1}{q^{d}-1}-\left(\alpha+q^{a+(n-1) d}\right) \frac{q^{n d}-1}{q^{d}-1} \\
& a_{n}=-q^{a+(2 n-1) d}\left(q^{(n-1) d}-1\right)\left(\alpha+q^{a+(n-2) d}\right) \\
& f_{n}=x^{n} \cdot \frac{q^{n d}-1}{q^{d}-1}\left(\alpha+q^{a+(n-1) d}\right) x^{n-1}+\frac{\left(q^{n d}-1\right)\left(q^{(n-1) d}-1\right)}{\left(q^{d}-1\right)\left(q^{2 d}-1\right)}\left(\alpha+q^{a+(n-2) d}\right) \\
& \quad \times\left(\alpha+q^{a+(n-1) d}\right) q^{d} x^{n-2}-\frac{\left(q^{n d}-1\right)\left(q^{(n-1) d}-1\right)\left(q^{(n-2) d}-1\right)}{\left(q^{d}-1\right)\left(q^{2 d}-1\right)\left(q^{8 d}-1\right)} \\
& \quad \times\left(\alpha+q^{a+(n-3) d}\right)\left(\alpha-q^{a+(n-2) d}\right)\left(\alpha+q^{a+(n-1) d}\right) q^{\frac{2 \times 3}{2} d} x^{n-2}+\ldots \\
& \quad \ldots+(-1)^{n}\left(\alpha+q^{a}\right) \ldots\left(\alpha+q^{a+(n-1) d}\right) q^{\frac{(n-1) n}{2} d} .
\end{aligned}
$$


Proof :-

Performing on $L_{4}$ the operations

(1) row $_{4}+\alpha\left(q^{2 d}-1\right)$ row $_{3}=q^{2 l}$ row $_{4}^{\prime}$ row $_{3}+\alpha\left(q^{d}-1\right)$ row $_{2}=q^{d}$ row $_{3}^{\prime}$

(2) $\operatorname{row}_{4}{ }^{\prime}+\alpha\left(q^{\alpha}-1\right)$ row $_{3}{ }^{\prime}=q^{\text {la }}$ row $_{4}{ }^{\prime \prime}$,

we get $\quad L_{4}=\left(\alpha+q^{a}\right)^{3}\left(\alpha+q^{a+d}\right)^{2}\left(\alpha+q^{a+2 d}\right) q^{2 d}\left(q^{d}\right)^{2}$

$$
\begin{gathered}
\times\left|\begin{array}{cccc}
1 & 1 & 1 & 1 \\
\alpha+q^{a+3 d} & \alpha+q^{a+2 d} & \alpha+q^{a+d} & \alpha+q^{a} \\
\left(\alpha+q^{a+3 d}\right)^{2} & \left(\alpha+q^{a+2 d}\right)^{2} & \left(\alpha+q^{a+d}\right)^{2} & \left(\alpha+q^{a}\right)^{2} \\
\left(\alpha+q^{a+3 l}\right)^{3} & \left(\alpha+q^{a+2 l}\right)^{3} & \left(\alpha+q^{a+d}\right)^{3} & \left(\alpha+q^{a}\right)^{3}
\end{array}\right| \\
L_{n}=(-1)^{\frac{n(n-1)}{2}}\left(\alpha+q^{a}\right)^{n-1}\left(\alpha+q^{a+d}\right)^{n-2} \ldots\left(\alpha+q^{a+(n-2) d}\right) q^{\frac{(n-2)(n-1) n}{3} d+\frac{(n-1) n}{2} a} \\
\times\left(q^{a}-1\right)^{n-1}\left(q^{2 d}-1\right)^{n-2} \ldots\left(q^{(n-1) a}-1\right) .
\end{gathered}
$$

(M).

In case (M) the formulae will be shown to be

$$
\begin{aligned}
& a_{1}=\frac{1}{\Pi\left(\alpha+q^{a}, \alpha+q^{\alpha+(r-1) d}\right)} \\
& b_{n}=\frac{q^{(n-1) d}-1}{q^{d}-1} \frac{\alpha+q^{a+(n-2) d}}{\alpha+q^{a+(r+2 u-4) d}}-\frac{q^{n d}-1}{q^{\alpha}-1} \frac{\alpha+q^{a+(n-1) d}}{\alpha+q^{a+(r+2 n-2) d}} \\
& a_{n}=\frac{\alpha q^{a+(2 n-4) d}\left(q^{(n-1) d}-1\right)\left(q^{r+(n-2) d}-1\right)\left(\alpha+q^{a+(n-2) d}\right)\left(\alpha+q^{\alpha+(r+n-3) d}\right)}{\left(\alpha+q^{a+(r+2 n-3) d}\right)\left(\alpha+q^{a+(r+2 n-4) d}\right)^{2}\left(\alpha+q^{a+(r+2 n-5) d}\right)} \\
& f_{n}=x^{n}-\frac{q^{n d}-1}{q^{d}-1} \frac{\alpha+q^{a+(n-1) d}}{\alpha+q^{a+(r+2) n-2) d}} \frac{q^{\frac{(n-1) n}{2} d}}{q^{\frac{(n-1) n}{2}} d} x^{n-1}+\frac{\left(q^{n d}-1\right)\left(q^{(n-1) d}-1\right)}{\left(q^{d}-1\right)\left(q^{2 d}-1\right)}
\end{aligned}
$$

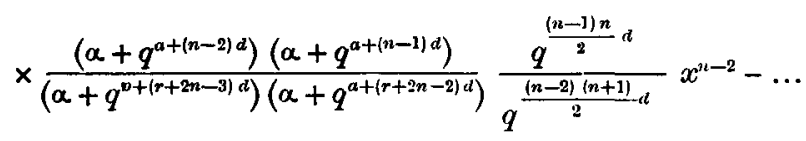

$$
\begin{aligned}
& \ldots+(-1)^{n} \frac{(\alpha+q)^{a}\left(\alpha+q^{\alpha+a}\right) \ldots\left(\alpha+q^{a+(n-1) d}\right)}{\left(\alpha+q^{a+(r+n-1) d}\right)\left(\alpha+q^{a+(r+n) d}\right) \ldots\left(\alpha+q^{a+(r+2 n-2) d}\right)} \frac{q^{\mathfrak{f}(n-1) n d}}{1} .
\end{aligned}
$$




\section{Proof :-}

First we are to perform on $M_{4}$ the operations

$$
\begin{aligned}
& \text { row }_{4}-\text { row }_{3}=\left(1-q^{r d}\right) \text { row }_{4}^{\prime} \\
& -q^{\text {rd }} \text { row }_{4}^{\prime}+\text { row }_{3}=\alpha \text { row }_{4}^{\prime \prime} \\
& \operatorname{row}_{3}-\operatorname{row}_{2}=\left(1-q^{\text {rd }}\right) \text { row }_{3}^{\prime} \\
& -q^{\text {rl }} \text { row }_{3}^{\prime}+\text { row }_{2}=\alpha \text { row }_{3}{ }^{\prime \prime} \\
& \text { row }_{2}-\text { row }_{1}=\left(1-q^{\text {rd }}\right) \text { row }_{2}^{\prime} \\
& -q^{\text {rd }} \text { row }_{2}^{\prime}+\text { row }_{1}=\alpha \text { row }_{2}^{\prime \prime} \\
& \text { row } \text { row }_{4} \text { - row }=\left(1-q^{(r+1) d}\right) \text { row }_{4}^{\prime \prime} \\
& -q^{(r+1) a} \text { row }_{4}^{\prime \prime \prime}+\text { row }_{3}^{\prime \prime}=a \text { row }_{4}^{1 v} \\
& \operatorname{row}_{3}^{\prime \prime}-\operatorname{row}_{2}^{\prime \prime}=\left(1-q^{(r+1) d}\right) \text { row }_{3}^{\prime \prime \prime} \\
& -q^{(r+1) d} \text { row }_{3}^{\prime \prime \prime}+\text { row }_{2}^{\prime \prime}=a \text { row }_{3}^{1 \mathrm{v}} \\
& \text { row }_{4}^{1 \mathrm{lv}}-\text { row }_{3}^{1 \mathrm{v}}=\left(1-q^{(r+2) a}\right) \text { row }_{4}{ }^{\mathrm{v}} \\
& -q^{(r+2) c l} \text { row }_{4}{ }^{v}+\text { row }_{3}{ }^{\text {iv }}=\alpha \text { row }_{4}{ }^{v} \text {. }
\end{aligned}
$$

In this way we get

$$
M_{4}=\frac{\left(q^{r d}-1\right)^{2}}{q^{3 r d}} \times \frac{\left(q^{(r+1) d}-1\right)^{2}}{q^{2(r+1) d}} \times \frac{\left(q^{(r+2) d}-1\right)}{q^{(r+2) d}} \times a^{3+2+1}
$$

multiplied by another determinant of order 4 .

If on this determinant we perform the operations

$$
\begin{aligned}
& \text { (1) } a\left(q^{d}-1\right) \text { row }_{4}+\text { row }_{3}=q^{d} \text { row }_{4}^{\prime} \\
& \alpha\left(q^{2 d}-1\right) \text { row }_{4}^{\prime}+\text { row }_{2}=q^{2 d} \text { row }_{4}^{\prime \prime} \\
& \alpha\left(q^{3 d}-1\right) \text { row }_{4}{ }^{\prime \prime}+\text { row }_{1}=q^{3 d} \text { row }_{4}^{\prime \prime \prime} \\
& \text { (2) } a\left(q^{d}-1\right) \operatorname{row}_{3}+\operatorname{row}_{2}=q^{d} \text { row }_{3}^{\prime} \\
& \alpha\left(q^{2 d}-1\right) \text { row }_{3}^{\prime}+\text { row }_{1}=q^{2 d} \text { row }_{3}^{\prime \prime} \\
& \text { (3) } a\left(q^{d}-1\right) \text { row }_{2}+\text { row }_{1}=q^{d} \text { row }_{2}^{\prime} \text {, }
\end{aligned}
$$

we get another determinant which, on putting $a$ for $a+(r-1) d$, becomes $F_{4}$.

$$
\begin{aligned}
M_{n}= & \frac{\left(q^{r l}-1\right)^{n-1}\left(q^{(r+1) d}-1\right)^{n-2} \ldots\left(q^{(r+n-2) d}-1\right)}{\left(q^{d}-1\right)^{n-1}\left(q^{2 d}-1\right)^{n-2} \ldots\left(q^{(n-1) d}-1\right)} \\
& \times q^{\frac{(n-1) n(n+1) d}{6}} \times q^{-a}\left\{\frac{(r+n-2)(r+n-1)(r+n)-(r-1) r(r+1)}{6}-\frac{(n-1) r(r-1) d}{2}\right\} \\
& \times \frac{1}{\Pi\left(\alpha+q^{a}, \alpha+q^{a+(r-2) d}\right) \ldots \Pi\left(\alpha+q^{a+(n-1) d}, \alpha+q^{a+(r+n-2) d}\right)} F_{n}^{\prime} .
\end{aligned}
$$




\section{PART II.}

In Part II. we shall consider cases in which a series of the form

$$
1+a_{0} x+\alpha_{1} x^{2}+a_{2} x^{3}+\ldots
$$

is to be converted into a continued fraction of the form

$$
1+\frac{a_{1} x}{1}+\frac{a_{2} x}{1}+\frac{a_{3} x}{1}+\cdots
$$

The reduction of this problem to a problem in determinants is effected by the formulae

$$
\begin{aligned}
a_{1} & =a_{0} \\
a_{2 n} & =-\frac{K_{n-1}}{K_{n}} \frac{{ }^{n} K_{n}}{{ }^{n-1} K_{n-1}} \\
a_{2 n+1} & =\frac{K_{n+1}}{K_{n}} \frac{{ }^{n-1} K_{n-1}}{{ }^{n} K_{n}}
\end{aligned}
$$

where the $K^{\prime}$ 's are the determinants previously introduced. These formulae are due to Heilermann, loc. cit., and are closely connected with the formulue used in Purt I.

(A).

Applying these formulae to case (A), we readily find

$$
\begin{aligned}
a_{1} & =\frac{1}{a} \\
a_{2 n} & =-\frac{\{a+(n-1) d\}^{2}}{\{a+(2 n-2) d\}\{a+(2 n-1) d\}} \\
a_{2 n+1} & =\frac{(n d)^{2}}{\{a+(2 n-1) d\}\{a+2 n d\}} .
\end{aligned}
$$

Ex. 1.-The series

$$
x \arctan x=\frac{x^{2}}{1}-\frac{x^{4}}{3}+\frac{x^{6}}{5}-\cdots
$$

gives Lagrange's continued fraction *

$$
\arctan x=\frac{x}{1}+\frac{x^{2}}{3}+\frac{(2 x)^{2}}{5}+\frac{(3 x)^{2}}{7}+\cdots
$$

* Euvres de Lagrange, 4, p. 323. 


\section{8}

Ex. 2.- $\int_{x}^{\infty} \frac{x^{a-1}}{1+x} d x=x^{a}\left(\frac{1}{1-a} \frac{1}{x}-\frac{1}{2-a} \frac{1}{x^{2}}+\frac{1}{3-a} \frac{1}{x^{3}}-\cdots\right)$

$$
=\frac{x^{a}}{(1-a) x}+\frac{(1-a)^{2}}{2-a}+\frac{1^{2}}{(3-a) x}+\frac{(2-a)^{2}}{4-a}+\frac{2^{2}}{(5-a) x}+\cdots
$$

$$
(\alpha<1 .)
$$

Ex. 3. $-\int_{0}^{\infty} \frac{x^{a-1}}{1+x} d x=x^{a-1}\left(\frac{x}{a}-\frac{x^{2}}{a+1}+\frac{x^{3}}{a+2}-\frac{x^{4}}{a+3}+\cdots\right)$

$$
=\frac{x^{a}}{a+}+\frac{a^{2} x}{a+1}+\frac{1^{2} x}{a+2}+\frac{(a+1)^{2} x}{a+3+\frac{2^{2} x}{a+4}+\cdots}
$$

(where $a$ is not negative.)

$$
\begin{aligned}
\text { LEx. 4. }-\int_{0}^{x} \frac{d x}{1+x^{r}}{ }^{*}=x^{1-r}\left\{\frac{x^{r}}{1}-\frac{x^{2 r}}{1+r}+\frac{x^{3 r}}{1+2 r}-\cdots\right\} \\
=\frac{x}{1+} \frac{1^{2}}{r+1}+\frac{r^{2} x^{r}}{2 r+1}+\frac{(r+1)^{2}}{3 r+1}+\frac{(2 r)^{2} x^{r}}{4 r+1}+\frac{(2 r+1)^{2} x^{r}}{5 r+1}+\cdots
\end{aligned}
$$

\section{Here}

$$
\begin{aligned}
a_{1} & =\frac{1}{a} \\
a_{2 n} & =-\frac{a+(n-1) d}{\{a+(2 n-2) d\}\{a+(2 n-1) d\}} \\
a_{2 n+1} & =\frac{n d}{\{a+(2 n-1) d\}\{a+2 n d\}} .
\end{aligned}
$$

$E x \cdot-a^{x}=1+\frac{x \log a}{1}-\frac{x \log a}{2}+\frac{x \log a}{3}-\frac{x \log a}{2}+\frac{x \log a}{5}-\frac{x \log a}{2}+\cdots$

Here

$$
\begin{aligned}
a_{1} & =\frac{D}{a} \\
a_{2 n} & =-(D+n d) \frac{a+(n-1) d}{\{a+(2 n-2) d\}\{a+(2 n-1) d\}} \\
a_{2 n+1} & =\frac{\{D-a-(n-1) d\} n d}{\{a+(2 n-1) d\}(a+2 n d)} .
\end{aligned}
$$

\footnotetext{
* Works of Lagrange, 4, p. 322.
} 
Ex. 1. $-\int_{l}^{m} \frac{(l-s)^{D}(s-m)^{q}}{x-8} d s=\frac{(l-m)^{D+q} \Gamma(q+1) \Gamma(D)}{\Gamma(q+D+1)}$

$$
\begin{gathered}
\times\left[\frac{D}{q+D+1} \frac{l-m}{l-x}+\frac{D(D+1)}{(q+D+1)(q+D+2)}\left(\frac{l-m}{l-x}\right)^{2}+\cdots\right] \\
a_{2 n}=\frac{(D+n)(q+D+n)}{(q+D+2 n-1)(q+D+2 n)} \\
a_{2 n+1}=-\frac{(n+q) n}{(q+D+2 n)(q+D+2 n+1)} .
\end{gathered}
$$

Exx. 2. $-\frac{\Gamma(a)}{\Gamma(D) \Gamma(a-D)} \int_{0}^{1} \frac{t^{D-1}(1-t)^{a-D-1}}{1-x t} d t=1+\frac{D}{a} x+\frac{D(D+1)}{a(a+1)} x^{2}+\cdots$

$$
=1+\frac{D x}{a}-\frac{(D+1) a x}{a+1}-\frac{1 \cdot(a-D) x}{a+2}-\frac{(D+2)(a+1) x}{a+3}-
$$

$$
\frac{2(a+1-D) x}{a+4}-\frac{(D+3)(a+2) x}{a+5}-\cdots
$$

Exx. 3.-For the series

$$
\begin{gathered}
\frac{D+(r-1) d}{a+(p-1) d} x+\frac{\{D+(r-1) d\}(D+r d)}{\{a+(p-1) d\}(a+p d)} x^{2}+\cdots \\
a_{2 n}=-\frac{\{D+(r+n-1) d\}\{a+(p+n-2) d\}}{\{a+(p+2 n-3) d\}\{a+(p+2 n-2) d\}} \\
a_{2 n+1}=-\frac{\{a-D+(p-r+n-1) d\} n d}{\{a+(p+2 n-2) d\}\{a+(p+2 n-1) d\}} .
\end{gathered}
$$

$\therefore \mathrm{II}(a, a+p-2) \int_{0}^{x} d x \int_{0}^{x} d x \ldots \int_{0}^{x} d x \int_{0}^{x} x^{a-1} d x \int_{0}^{1} \frac{t^{D-1}(1-t)^{a-D-1}}{1-x t} d t$

$$
=\frac{x^{a+p-1}}{a+p-1}-\frac{D(a+p-1) x}{a+p}-\frac{1 \cdot(a-D+p) x}{a+p+1}-\frac{(D+1)(a+p) x}{a+p+2}-\cdots
$$

The number of signs of integration is $p+1$.

Here

(E).

$$
\begin{aligned}
a_{2 n} & =-\frac{\{a+(n-1) d\}\{a+(n+r-2) d\}}{\{a+(2 n+r-3) d\}\{a+(2 n+r-2) d\}} \\
a_{2 n+1} & =-\frac{n d^{2}(r+n-1)}{\{a+(2 n+r-2) d\}\{a+(2 n+r-1) d\}}
\end{aligned}
$$

Ex. 1. $-\Pi(a, a+r-2) \int_{0}^{x} d x \ldots \int_{0}^{x} d x \int_{0}^{x} \frac{x^{a-1}}{1-x} d x$

$$
=\frac{x^{a+r-1}}{a+r-1}-\frac{a(a+r-1) x}{a+r} \frac{1 \cdot r x}{a+r+1}-\frac{(a+1)(a+r) x}{a+r+2-} \frac{2(r+1) x}{a+r+3}-\ldots
$$

The number of signs of integration is $r$. 
Ex. $2 .-(r-1) ! \int_{0}^{x} d x \ldots \int_{0}^{x} d x \int_{0}^{x}-\log (1-x) d x$ $=\frac{x^{r}}{r}-\frac{1 \cdot r x}{r+1}-\frac{1 \cdot r x}{r+2}-\frac{2(r+1) x}{r+3}-\frac{2(r+1) x}{r+4}-\frac{3(r+2) x}{r+5}-\frac{3(r+2) x}{r+6}-\cdots$

The number of signs of integration is $(r-1)$.

Ex. 3. $-\{1$. 3. $5 \ldots(2 r-3)\} \int_{0}^{x} x d x \ldots \int_{0}^{x} x d x \int_{0}^{x} x \tan ^{-1} x d x$ $=\frac{x^{2 r-1}}{2 r-1}+\frac{1 \cdot(2 r-1) x^{2}}{2 r+1+\frac{4 \cdot 1 r x^{2}}{2 r+3}}+\frac{3(2 r+1) x^{2}}{2 r+5}+\frac{4 \cdot 2(r+1) x^{2}}{2 r+7}+\cdots$

(F).

Here

$$
\begin{aligned}
a_{2 n} & =-\frac{\left(\alpha+q^{a+(n-1) d}\right)^{2}}{\left(\alpha+q^{a+(2 n-1) d}\right)\left(\alpha+q^{a+(2 n-2) d}\right)} q^{(n-1) d} \\
a_{2 n+1} & =\frac{\alpha\left(q^{n d}-1\right)^{2}}{\left(\alpha+q^{a+2 n d}\right)\left(\alpha+q^{a+(2 n-1) d}\right)} q^{a+(n-1) a} .
\end{aligned}
$$

$(\mathbf{G})$.

Here

$$
\begin{aligned}
a_{2 n} & =\frac{\alpha+q^{a+(n-1) d}}{\left(\alpha+q^{a+(2 n-1) d}\right)\left(\alpha+q^{a+(2 n-2) d}\right)} q^{(n-1) d} \\
a_{2 n+1} & =\frac{q^{n d}-1}{\left(\alpha+q^{a+2 n d}\right)\left(\alpha+q^{a+(2 n-1) d}\right)} q^{a+(2 n-1) d}
\end{aligned}
$$

Here

$$
\begin{aligned}
a_{2 n} & =\frac{\left(\alpha+q^{D+n d}\right)\left(\alpha+q^{a+(n-1) d}\right)}{\left(\alpha+q^{a+(2 n-1) d}\right)\left(\alpha+q^{a+(2 n-2) d}\right)} q^{(n-1) d} \\
a_{2 n+1} & =-\frac{\alpha\left(q^{D}-q^{a+(n-1) d}\right)\left(q^{n d}-1\right)}{\left(\alpha+q^{a+2 n d}\right)\left(\alpha+q^{a+(2 n-2) d}\right)} q^{n d}
\end{aligned}
$$

(L).

Here

$$
\begin{aligned}
a_{2 n} & =-\left(\alpha+q^{a+(n-1) d}\right) q^{(n-1) d} \\
a_{2 n+1} & =-q^{a+(n-1) d}\left(q^{n d}-1\right) .
\end{aligned}
$$


(M).

Here

$$
\begin{aligned}
a_{2 n} & =-\frac{\left(a+q^{a+(n-1) d}\right)\left(a+q^{a+(r+n-2) d}\right)}{\left(a+q^{a+(r+2 n-2) d}\right)\left(a+q^{a+(r+2 n-3} d\right)} q^{(n-1) d} \\
a_{2 n+1} & =\frac{a\left(q^{n a}-1\right)\left(q^{r+(n-1) d}-1\right) q^{a+(n-1) d}}{\left(a+q^{a+(r+2 n-1) d}\right)\left(a+q^{a+(r+2 n-2) d}\right)} .
\end{aligned}
$$

Here

$(\mathrm{N})$.

Proof :-

$$
\begin{aligned}
a_{1} & =q \\
a_{2 n} & =-q^{4 n-1} \\
a_{2 n+1} & =q^{2 n+1}\left(1-q^{2 n}\right) .
\end{aligned}
$$

$$
\begin{aligned}
& N_{4}=q^{1+2^{2}+3^{2}+4^{2}}\left|\begin{array}{cccc}
1 & 1 & 1 & 1 \\
q^{3} & q^{5} & q^{7} & q^{9} \\
q^{8} & q^{12} & q^{18} & q^{20} \\
q^{15} & q^{21} & q^{27} & q^{33}
\end{array}\right| \\
& =q^{1+2^{2}+8^{2}+4^{2}}\left(q^{2}-1\right)\left(q^{4}-1\right)\left(q^{6}-1\right)\left|\begin{array}{ccc}
q^{3} & q^{5} & q^{7} \\
q^{8} & q^{13} & q^{16} \\
q^{15} & q^{21} & q^{27}
\end{array}\right| \\
& =q^{1+2^{2}+3^{3}+4^{2}}\left(q^{2}-1\right)\left(q^{4}-1\right)\left(q^{6}-1\right) q^{2+1+6}\left|\begin{array}{ccc}
q & q^{3} & q^{5} \\
q^{4} & q^{8} & q^{12} \\
q^{3} & q^{15} & q^{23}
\end{array}\right| \\
& =q^{1+3^{2}+3^{2}+4^{2}}\left(q^{2}-1\right)\left(q^{4}-1\right)\left(q^{6}-1\right) \frac{q^{2+4+6}}{q^{2^{2}+2^{2}}} N_{3} \text {. }
\end{aligned}
$$

\section{Similarly,}

$$
\begin{aligned}
\frac{N_{n}}{N_{n-1}} & =q^{(n-1)^{2}+n(2 n-1)}\left(q^{2}-1\right)\left(q^{4}-1\right)\left(q^{6}-1\right) \ldots\left\{q^{2(n-1)}-1\right\} \\
{ }^{4} N_{4} & =q^{2^{2}+3^{2}+4^{2}+3^{2}}\left|\begin{array}{cccc}
1 & 1 & 1 & 1 \\
q^{5} & q^{7} & q^{9} & q^{11} \\
q^{13} & q^{16} & q^{20} & q^{24} \\
q^{21} & q^{2 \pi} & q^{33} & q^{39}
\end{array}\right| \\
& =q^{2^{2}+3^{2}+4^{2}+5^{2}}\left(q^{2}-1\right)\left(q^{4}-1\right)\left(q^{6}-1\right)\left|\begin{array}{lll}
q^{5} & q^{7} & q^{9} \\
q^{12} & q^{16} & q^{20} \\
q^{21} & q^{2 \pi} & q^{33}
\end{array}\right|
\end{aligned}
$$




$$
\begin{aligned}
& =q^{2^{2}+3^{2}+4^{2}+5^{2}}\left(q^{2}-1\right)\left(q^{4}-1\right)\left(q^{6}-1\right) q^{3+4+6}\left|\begin{array}{ccc}
q^{3} & q^{5} & q^{7} \\
q^{8} & q^{12} & q^{16} \\
q^{15} & q^{21} & q^{27}
\end{array}\right| \\
& =q^{2^{2}+3^{2}+4^{2}+5^{2}}\left(q^{2}-1\right)\left(q^{4}-1\right)\left(q^{6}-1\right) \frac{q^{2+4+6}}{{q^{1{ }^{2}+2^{2}+3^{2}}}^{3} N_{3} .}
\end{aligned}
$$

\section{Similarly,}

$$
\frac{{ }^{n} N_{n}}{N_{n}}=q^{n(n+2)+n(2 n-1)}\left(q^{2}-1\right)\left(q^{4}-1\right) \ldots\left\{q^{2(n-1)}-1\right\} .
$$

Thus we get

$$
\begin{aligned}
a_{2 n} & =-\frac{N_{n-1}}{N_{n}} \times \frac{n N_{n}}{n-1} N_{n-1}=-q^{\mathrm{d} n-1} \\
a_{3_{n+1}} & =-\frac{N_{n+1}}{N_{n}} \times \frac{{ }^{n-1} N_{n-1}}{{ }^{n} N_{n}}=q^{2 n+1}\left(1-q^{2 n}\right) .
\end{aligned}
$$

We have therefore

$$
\begin{gathered}
q x+q^{4} x+q^{9} x+\ldots+q^{2^{2}} x+\ldots \\
=\frac{q x}{1}-\frac{q^{3} x}{1}+\frac{q^{3}\left(1-q^{2}\right) x}{1}-\frac{q^{7} x}{1}+\frac{q^{5}\left(1-q^{4}\right) x}{1}-\frac{q^{11} x}{1}+\frac{q^{7}\left(1-q^{6}\right) x}{1}-\ldots
\end{gathered}
$$

a result first given by Eisenstein.

In conclusion, I must thank Professor Whittaker, who introduced me to the subject, and under whose guidance $I$ have been enabled to prepare this paper. 